\title{
Osteoblast-derived WISP-1 increases VCAM-1 expression and enhances prostate cancer metastasis by down-regulating miR- 126
}

\author{
Huai-Ching Tai ${ }^{1}$, An-Chen Chang ${ }^{2}$, Hong-Jeng $\mathbf{Y u}^{1}$, Chao-Yuan Huang ${ }^{1}$, Yu-Chieh \\ Tsai $^{3}$, Yu-Wei Lai ${ }^{4}$, Hui-Lung Sun ${ }^{5}$, Chih-Hsin Tang ${ }^{2,6,7}$ and Shih-Wei Wang ${ }^{8}$ \\ ${ }^{1}$ Department of Urology, National Taiwan University Hospital, Taipei, Taiwan \\ ${ }^{2}$ Graduate Institute of Basic Medical Science, China Medical University, Taichung, Taiwan \\ ${ }^{3}$ Department of Oncology, National Taiwan University Hospital, Taipei, Taiwan \\ ${ }^{4}$ Division of Urology, Taipei City Hospital Renai Branch, Taipei, Taiwan \\ ${ }^{5}$ Department of Molecular Virology, Immunology and Mediccal Genetics, Ohio state University, Columbus, OH, USA \\ ${ }^{6}$ Department of Pharmacology, School of Medicine, China Medical University, Taichung, Taiwan \\ 7 Department of Biotechnology, College of Health Science, Asia University, Taichung, Taiwan \\ ${ }^{8}$ Department of Medicine, Mackay Medical College, New Taipei City, Taiwan \\ Correspondence to: Shih-Wei Wang, email: shihwei@mmc.edu.tw
}

Chih-Hsin Tang, email: chtang@mail.cmu.edu.tw

Keywords: WISP-1; Osteoblasts; Prostate cancer; miR-126; VCAM-1

Received: May 29, $2014 \quad$ Accepted: July 29, $2014 \quad$ Published: July 30, 2014

This is an open-access article distributed under the terms of the Creative Commons Attribution License, which permits unrestricted use, distribution, and reproduction in any medium, provided the original author and source are credited.

\section{ABSTRACT}

Bone metastases of prostate cancer ( $\mathrm{PCa}$ ) may cause intractable pain. Wntinduced secreted protein-1 (WISP-1) belongs to the CCN family (CTGF/CYR61/ NOV) that plays a key role in bone formation. We found that osteoblast-conditioned medium (ОВCM) stimulates migration and vascular cell adhesion molecule-1 (VCAM1) expression in human PCa (PC3 and DU145) cells. Osteoblast transfection with WISP-1 shRNA reduced OBCM-mediated PCa migration and VCAM-1 expression. Stimulation of PCa with OBCM or WISP-1 elevated focal adhesion kinase (FAK) and p38 phosphorylation. Either FAK and p38 inhibitors or siRNA abolished osteoblastderived WISP-1-induced migration and VCAM-1 expression. Osteoblast-derived WISP-1 inhibited miR-126 expression. Moreover, miR-216 mimic reversed the WISP1-enhanced migration and VCAM-1 expression. This study suggests that osteoblastderived WISP-1 promotes migration and VCAM-1 expression in human PCa cells by down-regulating miR-126 expression via av $\beta 1$ integrin, FAK, and p38 signaling pathways. Thus, WISP-1 may be a new molecular therapeutic target in PCa bone metastasis.

\section{INTRODUCTION}

Prostate cancer $(\mathrm{PCa})$ is the most commonly diagnosed malignancy in the United States and other Western countries [1]. During early stages, surgery is the most frequent therapeutic intervention. In advanced stages, however, systemic intervention is required to inhibit tumor growth and prevent secondary metastases. Bone metastasis is a common complication associated with advanced $\mathrm{PCa}$, often causing acute pain and bone fracture.
Bone metastasis has prognostic value in $\mathrm{PCa}$, since the extent of disease in the bone significantly affects survival [2-4]. Metastasis to the bone, involving the osteoblasts and osteoclasts, results in bone lesions [5]. Osteoblasts, the major cellular component of bones, play a key role in osteogenesis $[6,7]$. In the tumor microenvironment, cancer cells yield soluble factors to stimulate osteoblast activation, proliferation, and maturation. On the other hand, they secrete bone matrix and growth factors, which promote malignancy and osteoblastic bone metastasis $[8$, 
9]. Therefore, osteoblast-derived factors are crucial during bone metastasis.

Wnt1-induced secreted protein-1 (WISP-1) is a cysteine-rich protein belonging to the Cyr61, CTGF, Nov (CCN) family of matricellular proteins that have developmental functions and regulate bone formation $[10,11]$. CCN family proteins are mostly secreted and associated with the extracellular matrix (ECM), which has been demonstrated to play an important role in tumor development, including tumor survival, proliferation, migration, and invasion [12-14]. WISP-1 is reportedly expressed in developing breast tumors in transgenic mice [15]. Mounting evidence also suggests that WISP-1 enhances tumorigenesis and metastasis in many types of cancer $[12,16,17]$. This indicates that WISP-1 plays a vital role in cancer development and metastasis.

Several cell adhesion molecules secreted by cancer cells are involved in metastasis; e.g., integrin, cadherin, immunoglobulin superfamily [18, 19]. Vascular cell adhesion molecule-1 (VCAM-1 or CD106), a member of the immunoglobulin superfamily, is a transmembrane glycoprotein that mediates adhesion of lymphocytes or monocytes to the vascular endothelium [20]. Aberrant expression of VCAM-1 in cancer cells has been documented in preclinical models as well as patient samples of gastric cancer [21] and renal cell carcinoma [22]. Likewise, VCAM-1 has been indicated to regulate tumor progression and bone metastasis in glioblastoma and breast cancer [23-25]. It is unknown whether VCAM1 has any functional role in PCa metastasis to the bone.

Bone-derived growth factor and chemokines play central roles as trophic factors that attract breast, lung, and prostate cancer cells to bone tissue [26]. Understanding the mechanism of interactions between human $\mathrm{PCa}$ and osteoblasts is critical to devising therapies for $\mathrm{PCa}$ bone metastasis. We hypothesized that osteoblast-derived WISP-1 may regulate VCAM-1 expression and promote the migration of $\mathrm{PCa}$ cells. We found that osteoblastderived WISP-1 promoted PCa migration and VCAM1 expression by down-regulating miR-126 through the $\alpha v \beta 1$ integrin/focal adhesion kinase (FAK)/p38 signaling pathway.

\section{RESULTS}

\section{Osteoblast-derived WISP-1 promotes prostate cancer cell motility}

Bone-derived growth factors and chemokines reportedly promote tumor metastasis to the bone [7, $8,28]$. We hypothesized that osteoblasts are capable of regulating $\mathrm{PCa}$ metastasis to the bone and we examined the effect of OBCM on PCa cell motility via a Transwell assay. We found that migration and invasion of PCa cells
(DU145 and PC-3) was OBCM concentration-dependent (Fig. 1A-B). It is well established that osteoblasts can synthesize and secrete WISP-1, which plays an important role in bone formation and cell differentiation [11, 29]. To pinpoint the contribution of WISP-1 in OBCM to PCa motility, we used a monoclonal antibody (mAb) against WISP-1. Figure 1C-D shows that WISP-1 mAb blocked OBCM-induced migration and invasion in PCa cells. To corroborate this hypothesis, we stimulated $\mathrm{PCa}$ cells with WISP-1 and found that this significantly increased migration and invasion in PCa cells (Fig. 1E-F). To confirm the role of WISP-1 in OBCM, we transfected osteoblasts with control or WISP-1 shRNA, collecting and applying their OBCM to PCa cells. As shown in Fig. 1G, transfection of osteoblasts with WISP-1 shRNA reduced WISP-1 expression in OBCM. WISP-1 shRNA also antagonized $\mathrm{OBCM}$-mediated $\mathrm{PCa}$ migration and invasion (Fig. 1H-I). These results indicate that osteoblast-derived WISP-1 promotes migration and invasion in PCa cells.

\section{Osteoblast-derived WISP-1-directing prostate cancer migration involves VCAM-1 up-regulation through integrin $\alpha \mathrm{v} \beta 1$ receptor}

VCAM-1 reportedly mediates tumor bone metastasis [25]. We hypothesized that VCAM-1 is involved in osteoblast-derived WISP-1-directed PCa migration. Stimulation of PCa cells with OBCM or WISP-1 increased $V C A M-1$ mRNA expression in a concentration-dependent manner (Fig. 2A and C). Transfection of PCa cells with VCAM-1 siRNA markedly inhibited OBCM- or WISP1-induced migration (Fig. 2B and D). WISP-1 shRNA antagonized OBCM-mediated VCAM-1 expression (Fig. 2E-F). These data suggest that OBCM-derived WISP1 -induced $\mathrm{PCa}$ migration occurs via up-regulation of VCAM-1 expression. WISP-1 is known to affect cell function by binding to the cell-surface integrin receptor [30]. Incubation of PCa cells with OBCM increased mRNA expression of $\alpha \mathrm{v}$ and $\beta 1$ integrin (data not shown). Co-transfection of PCa cells with $\alpha v \beta 1$ siRNA markedly reduced OBCM- or WISP-1-enhanced cell migration (Fig. $3 \mathrm{~A}$ and $\mathrm{D})$. On the other hand, $\alpha v \beta 1$ siRNA diminished OBCM- or WISP-1-mediated VCAM-1 expression (Fig. 3B, C, E). Thus, osteoblast-derived WISP-1 increases migration and VCAM-1 expression in human PCa cells through integrin $\alpha v \beta 1$ receptor.

\section{FAK and p38 signal pathways are involved in osteoblast-derived WISP-1-mediated PCa migration and VCAM-1 expression}

FAK, a widely expressed non-receptor protein tyrosine kinase, is an early downstream factor of integrinmediated signaling that regulates cellular function [31]. To verify whether FAK activation is involved in 

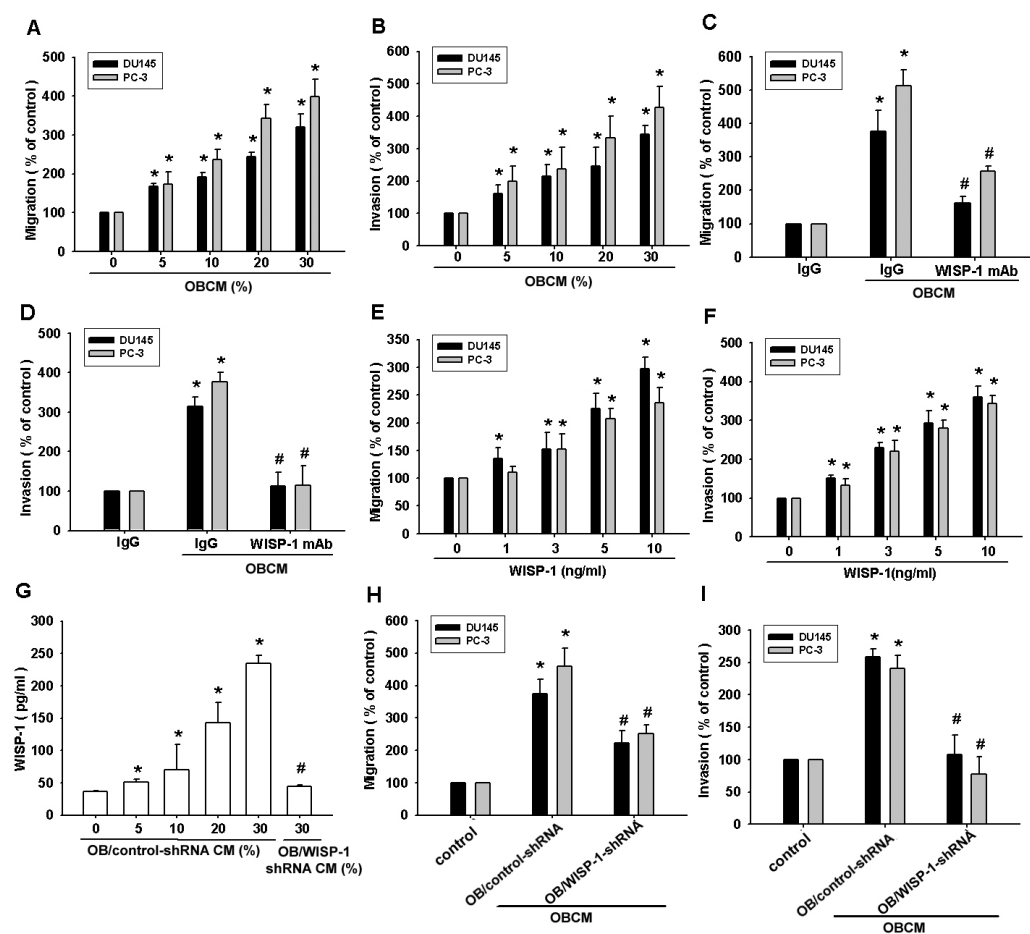

Fig.1: Osteoblast-derived WISP-1 increases prostate cancer motility. (A-D) Prostate cancer (PCa) cells (DU145 and PC3) were incubated with various concentrations of osteoblast conditional medium (OBCM) or OBCM pretreated with IgG or WISP-1 monoclonal antibody $(10 \mu \mathrm{g} / \mathrm{mL})$ for $30 \mathrm{~min}$; in vitro migration and invasion were measured by a Transwell assay. (E, F) PCa cells were incubated with WISP-1 $(1-10 \mathrm{ng} / \mathrm{mL})$ for $24 \mathrm{~h}$; in vitro migration and invasion were measured by a Transwell assay. (G-I) Osteoblasts were transfected with control or WISP-1 shRNA for $24 \mathrm{~h}$, the medium was collected as OBCM, and WISP-1 expression was examined by ELISA. OBCM was applied to PCa cells for $24 \mathrm{~h}$, and in vitro migration and invasion were measured by a Transwell assay. Results expressed as mean \pm SEM. *, $p<0.05$ compared with control; \#, $p<0.05$ compared with OBCM or WISP-1-treated group.
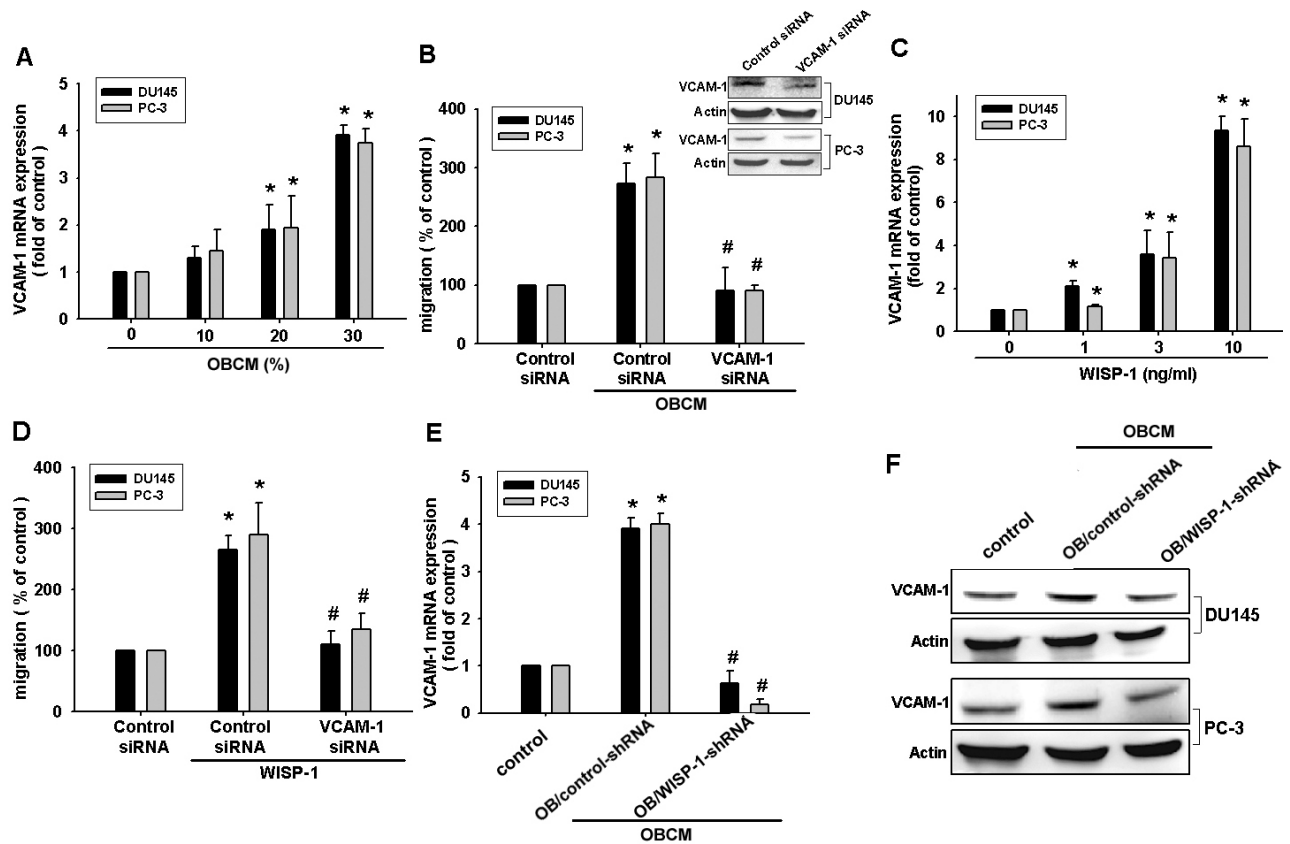

Fig.2: Vascular cell adhesion molecule-1 (VCAM-1) is involved in osteoblast-derived WISP-1-mediated PCa cell migration. (A, C) PCa cells were incubated with various OBCM or WISP-1 concentrations for $24 \mathrm{~h}$, and VCAM-1 expression was examined by real-time quantitative polymerase chain reaction (RT-qPCR). (B, D) PCa cells were transfected with VCAM-1 siRNA for 24 $\mathrm{h}$ followed by stimulation with OBCM (30\%) or WISP-1 (10 ng/mL) for $24 \mathrm{~h}$; in vitro migration was measured by a Transwell assay. (E, F) Osteoblasts were transfected with control or WISP-1 shRNA for $24 \mathrm{~h}$, and the medium was collected as OBCM and applied to PCa cells for $24 \mathrm{~h}$. VCAM-1 expression was examined by RT-qPCR and Western blot. Results are expressed as mean \pm SEM. *, $p<0.05$ compared with control; \#, $p<0.05$ compared with OBCM or WISP-1-treated group. 
osteoblast-derived WISP-1-induced cell migration, we directly measured phosphorylation of FAK in response to OBCM. Stimulation of PCa cells with OBCM increased FAK phosphorylation (Fig. 4A). In contrast, knockdown of WISP-1 in osteoblasts diminished OBCM-mediated FAK phosphorylation (Fig. 4A). FAK inhibitor or siRNA reduced OBCM- or WISP-1-increased cell migration and VCAM-1 expression in human PCa (Fig. 4C-H), indicating that WISP-1 enhanced FAK phosphorylation (Fig. 4B).

Mitogen-activated protein kinase (MAPK) activation is reported to be indispensible for migration in human $\mathrm{PCa}$ [32]. To delineate signal pathways downstream of WISP1, we examined MAPK activity in OBCM-treated cells. Fig. 4A demonstrated that OBCM exposure caused an increase in the phosphorylation of ERK, p38, and JNK. Knockdown of WISP-1 reduced OBCM-mediated p38 but not ERK and JNK phosphorylation, indicating that p38 (not ERK or JNK) is involved in osteoblast-derived WISP1-mediated cell function. Recombinant human WISP1 promoted p38 phosphorylation in a time-dependent manner (Fig. 4B). To test the involvement of p38 in osteoblast-derived WISP-1-mediated migration, we used p38 inhibitor (SB203580) and siRNA. We found that p38 inhibitor or siRNA reduced OBCM- or WISP-1-induced cell migration and VCAM-1 expression (Fig. 4C-H), suggesting that the FAK and p38 pathways are involved in osteoblast-derived WISP-1-mediated migration and VCAM-1 expression in human PCa.

Osteoblast-derived WISP-1 increased cell migration and VCAM-1 expression by downregulating miR-126 through $\alpha v \beta 1$ integrin, FAK, and p38 signaling pathways

It has been reported that miR-126, regulating VCAM-1 expression, is involved in many cellular functions [33-35]. We hypothesized that miR-126, regulating VCAM-1, mediates osteoblast-derived WISP1 -induced cell migration. We found that incubation of $\mathrm{PCa}$ cells with OBCM reduced miR-126 expression, while WISP-1 shRNA rescued OBCM-inhibited miR126 expression (Fig. 5A). Stimulation of PCa cells with WISP-1 inhibited miR-126 expression in a concentrationdependent manner (Fig. 5B). To confirm that miR-126 is involved in osteoblast-derived WISP-1-mediated cell migration, we used an miR-126 mimic and found that transfection with the miR-126 mimic inhibited OBCMinduced migration and VCAM-1 expression (Fig. 5C-D). Simultaneously, $\alpha v \beta 1$, FAK, and p38 siRNA reversed OBCM-inhibited miR-126 expression and promoter activity (Fig. 5E-F), indicating that osteoblast-derived WISP-1 suppresses miR-216 via the $\alpha v \beta 1 / F A K / p 38$
A

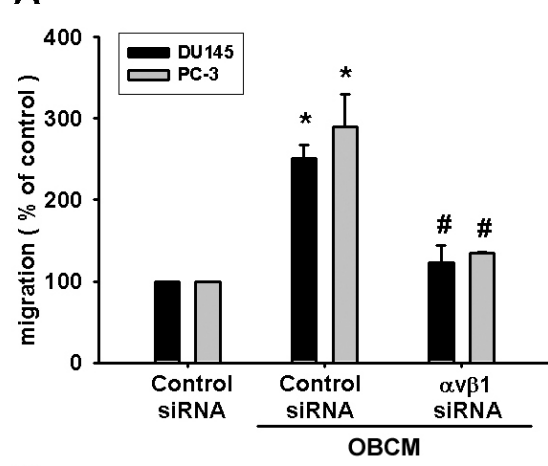

D

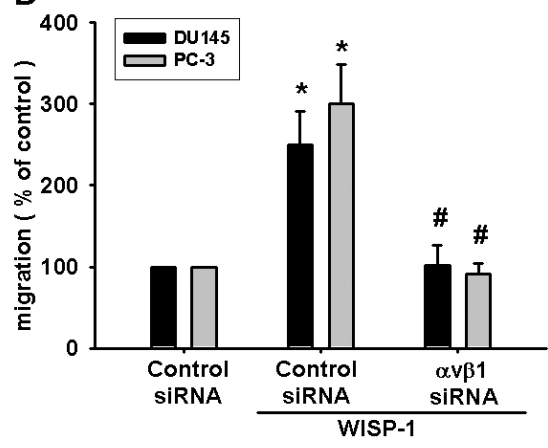

B

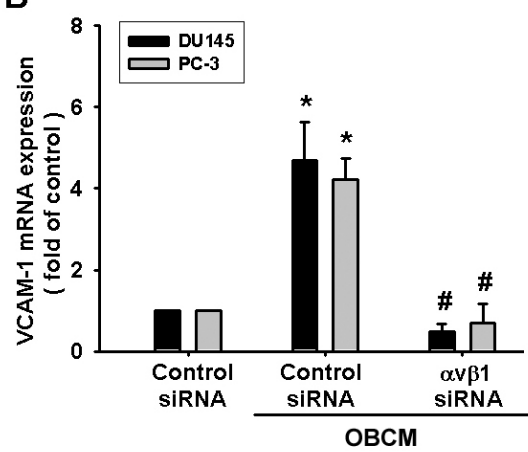

E

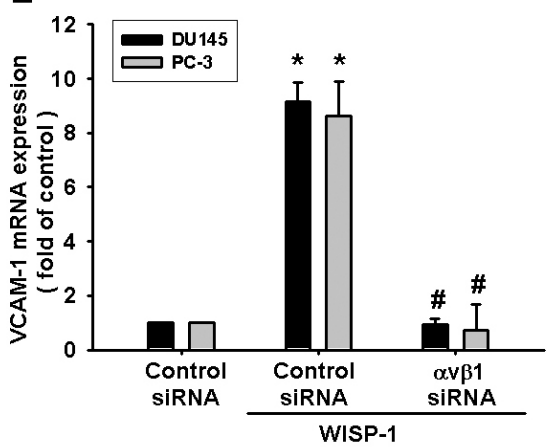

C

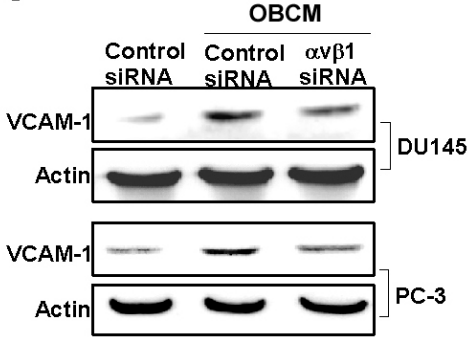

Fig.3: Osteoblast-derived WISP-1 boosts migration and VCAM-1 expression via integrin $\alpha$ v $\beta 1$ receptor. (A-E) PCa cells were transfected with $\alpha v \beta 1$ siRNA for $24 \mathrm{~h}$ followed by stimulation with OBCM (30 \%) or WISP-1 (10 ng/mL) for $24 \mathrm{~h}$; in vitro migration and VCAM-1 expression were determined by Transwell, RT-qPCR, and Western blot analyses. Results are expressed as mean \pm SEM. * $p$ $<0.05$ compared with control; \#, $p<0.05$ compared with OBCM or WISP-1-treated group. 

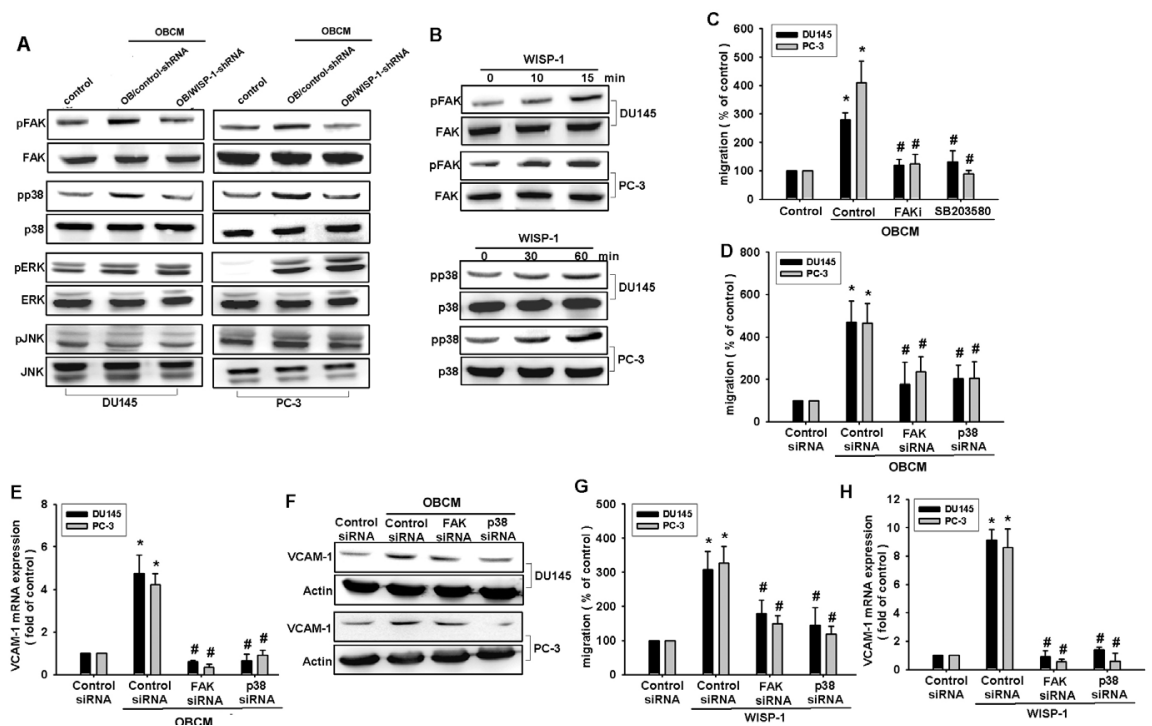

Fig.4: FAK and p38 pathways are involved in osteoblast-derived WISP-1-increased migration and VCAM-1 expression. (A) Osteoblasts were transfected with control or WISP-1 shRNA for $24 \mathrm{~h}$, and the medium was collected as OBCM and applied to PCa cells. FAK, p38, ERK, and JNK phosphorylation was examined by Western blot. (B) PCa cells were incubated with WISP-1 (10 ng/mL) for the indicated time intervals; FAK and p38 phosphorylation was examined by Western blot. (C-H) PCa cells were pretreated with FAK inhibitor $(10 \mu \mathrm{M})$ and SB203580 $(10 \mu \mathrm{M})$ or transfected with FAK and p38 siRNA for $24 \mathrm{~h}$ followed by stimulation with OBCM (30\%) or WISP-1 (10 ng/mL) for $24 \mathrm{~h}$; in vitro migration and VCAM-1 expression were measured by Transwell, RT-qPCR, and Western blot analyses. Results are expressed as mean \pm SEM. *,$p<0.05$ compared with control; \#, $p<0.05$ compared with OBCM or WISP-1-treated group.
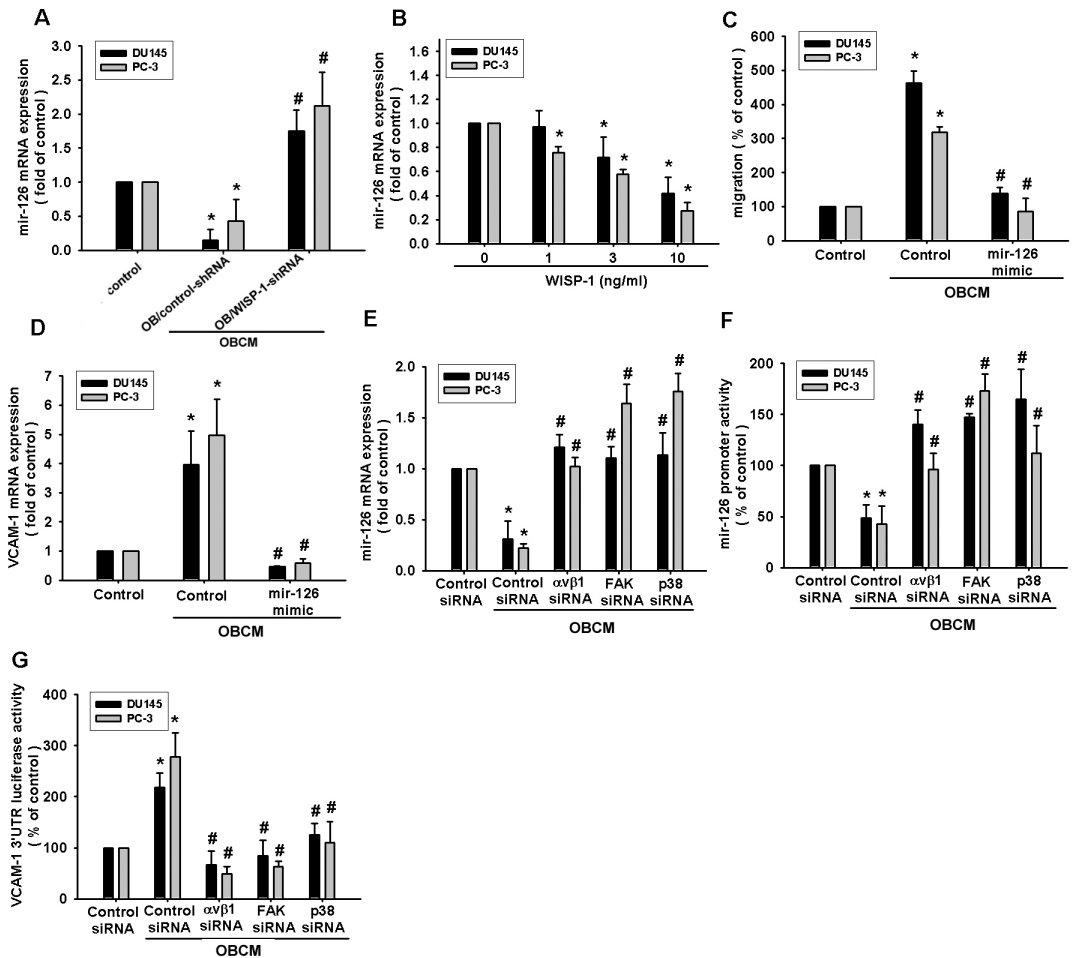

OBCM

Fig.5: Osteoblast-derived WISP-1 boosts PCa migration and VCAM-1 expression by down-regulating miR-126 expression. (A) Osteoblasts were transfected with control or WISP-1 shRNA for $24 \mathrm{~h}$. Medium was collected as OBCM and applied to PCa cells; miR-126 expression was examined by qPCR. (B) PCa cells were incubated with WISP-1 (10 ng/mL) for the indicated time intervals, and miR-126 expression was examined by qPCR. (C, D) PCa cells were transfected with an miR-126 mimic for $24 \mathrm{~h}$ followed by stimulation with OBCM (30\%) or WISP-1 (10 ng/mL) for $24 \mathrm{~h}$; miR-126 expression was examined by qPCR. (E-G) PCa cells were transfected with $\alpha v \beta 1$, FAK, or p38 siRNA for $24 \mathrm{~h}$ followed by stimulation with OBCM (30 \%) or WISP-1 (10 ng/mL) for $24 \mathrm{~h}$, and miR126 expression, miR-126 promoter activity, or VCAM-1 3'UTR activity were examined. Results are expressed as mean \pm SEM. *, $p<0.05$ compared with control; \#, $p<0.05$ compared with OBCM or WISP-1-treated group. 
pathway. To examine whether miR-216 regulates the 3'UTR of $V C A M-1$, we constructed a luciferase-reporter vector harboring the $3^{\prime} \mathrm{UTR}$ of $V C A M-1$ mRNA and another vector containing the miR-216-binding site. The data showed that OBCM increased luciferase activity in the VCAM-1 3'UTR plasmid, whereas $\alpha \mathrm{v} \beta 1$, FAK, or p38 siRNA reduced OBCM-mediated $V C A M-1$ 3'UTR activity (Fig. 5G). Taken together, these data demonstrate that miR-216 directly represses VCAM-1 protein expression via binding to the $3^{\prime} \mathrm{UTR}$ of human $V C A M-1$ through the $\alpha \mathrm{v} \beta 1 / \mathrm{FAK} / \mathrm{p} 38$ signaling pathway.

\section{DISCUSSION}

$\mathrm{PCa}$ cells exhibit a remarkable tendency to metastasize to the bone [36-39]. Analysis of trophic signals that control $\mathrm{PCa}$ bone metastasis is crucial to identify new molecular targets for anti-metastasis therapy. We hypothesized that osteoblast-derived factors help to direct migration of $\mathrm{PCa}$ cells and found that osteoblastderived factors induced migration of human $\mathrm{PCa}$ cells. Using WISP-1 shRNA to knock down WISP-1 expression in osteoblasts, we identified WISP-1 as the chief factor in osteoblasts that promotes PCa migration and VCAM1 up-regulation. One mechanism we found that underlies osteoblast-derived WISP-1-directed migration was transcriptional up-regulation of VCAM-1 expression by down-regulation of miR-126 through $\alpha v \beta 1$ integrin, FAK, and p38 signaling pathways.

Tumor invasion and metastasis are the main biological characteristics of cancer. Metastasis is the major cause of cancer-related death, involving multiple processes: invasion of cells and altering cellcell adhesion properties, rearrangement of the ECM environment, suppression of anoikis, and reorganization of the cell cytoskeleton [40]. Cell adhesion molecules are transmembrane glycoproteins that mediate cell-cell and cell-ECM interactions. VCAM-1, a cell adhesion molecule, reportedly mediates epithelial-to-mesenchymal transition, invasion, and bone metastasis [23, 24]. In breast cancer, VCAM-1 is a crucial activator of indolent bone metastasis and osteoclast recruitment to form bone lesions. Pretreatment with VCAM-1 antibody reduces breast cancer cell migration and bone metastasis [25], indicating that VCAM-1 plays a critical role in tumor migration; its disruption can prevent bone metastasis. Our study uncovered evidence of VCAM-1 as a major factor in osteoblast-derived WISP-1-mediated migration in human $\mathrm{PCa}$ cells. We found that osteoblast-derived WISP-1 induced PCa cells to express VCAM-1, whereas siRNA against VCAM-1 significantly reduced WISP-1-mediated cell motility. VCAM-1 is thus a downstream effector in the osteoblast-derived WISP-1-increased motility of human PCa cells.

Newly identified small noncoding RNAs, miRNAs, belong to a novel class of gene regulators that control gene expression by binding to complementary sequences in the 3'UTRs of target mRNAs [41, 42]. Dysregulated miRNA expression has been reported in human cancer and may affect multiple steps during metastasis [43]. miR-126 has been reported as a negative regulator of VCAM-1 that mediates several cellular functions [33-35]. Our study defines a mechanism for miR-126 function wherein miR126 mediates PCa migration by suppressing VCAM-1 expression. Our data show that osteoblast-derived WISP1 inhibited miR-126 expression and promoter activity. Transfection with an miR-126 mimic halted osteoblastderived WISP-1-mediated migration and VCAM-1 expression. Moreover, we showed that miR-126 directly repressed VCAM-1 protein expression through binding to the 3'-UTR of human VCAM-1, thereby negatively regulating VCAM-1-mediated metastasis.

Previous studies have shown that MAPK is activated after stimulation of $\mathrm{CCN}$ family proteins $[26,44]$. We found that OBCM promoted ERK, p38, and JNK phosphorylation. However, knockdown of WISP-1 in osteoblasts decreased OBCM-induced p38 phosphorylation, while other MAPKs (JNK and ERK) were unaffected. This suggests that $\mathrm{p} 38$, but not JNK and ERK, is involved in osteoblast-derived WISP1-mediated cell functions. In addition, p38 inhibitor reduced osteoblast-derived WISP-1-enhanced migration and VCAM-1 expression, as confirmed by inhibition of osteoblast-derived WISP-1-enhanced migration and

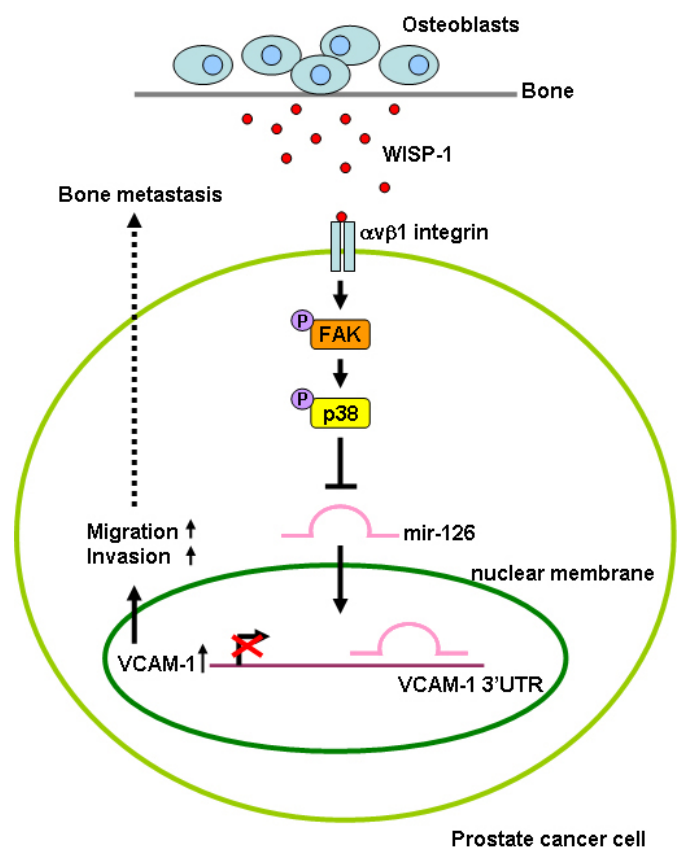

Fig.6: Schematic presentation of signaling pathways involved in osteoblast-derived WISP-1-induced migration and VCAM-1 expression of PCa cells. Osteoblast-derived WISP-1 promotes migration and VCAM-1 expression in human $\mathrm{PCa}$ cells by down-regulating miR-126 expression via $\alpha v \beta 1$ integrin, $\mathrm{FAK}$, and $\mathrm{p} 38$ signaling pathways. 
VCAM-1 expression in human PCa by p38 siRNA. Transfection with $\alpha v \beta 1$, FAK, or p38 siRNA diminished OBCM-mediated miR-126 expression and VCAM-1 3'UTR activity. Thus, our data indicate that $\alpha v \beta 1$ integrin, FAK, and p38 signaling pathways are involved in osteoblast-derived WISP-1-inhibited miR-126 expression in human PCa cells.

Bone is a common site of cancer metastasis. PCa shows a particular predilection for metastasis to the bone. Bone-derived growth factors and chemokines play central roles as trophic factors that attract PCa cells to bone tissue [3]. Osteoblast-derived factors constitutively secreted by human osteoblasts play a key role of hematopoietic cells in the marrow [15]. The effect of osteoblast-derived factors on VCAM-1 expression and migration activity in human PCa cells remains mostly unknown. We observed that osteoblast-derived WISP-1 promoted migration and VCAM-1 expression in human PCa cells by downregulating miR-126 expression via $\alpha v \beta 1$ integrin, FAK, and p38 signaling pathways. Inhibition of WISP-1 thus presents a new, plausible therapeutic target in prostate cancer-related bone metastasis.

\section{MATERIALS AND METHODS}

\section{Materials}

Anti-mouse and anti-rabbit IgG-conjugated horseradish peroxidase; rabbit polyclonal antibodies specific to VCAM-1, p-ERK, ERK, p-p38, p38, p-JNK, JNK, p-FAK, FAK, and $\beta$-actin; and WISP-1 short hairpin RNA (shRNA) and control shRNA plasmids were purchased from Santa Cruz Biotechnology (Santa Cruz, CA); p38 inhibitor (SB203580) was from Enzo Life Sciences (Farmingdale, NY); FAK inhibitor was from Calbiochem (San Diego, CA); recombinant human WISP1 was from R\&D Systems (Minneapolis, MN); Dulbecco's modified Eagle's medium (DMEM), RPMI-1640 medium, fetal bovine serum (FBS), and all other culture reagents were obtained from Gibco-BRL Life Technologies (Grand Island, NY); the luciferase assay kit was from Promega (Madison, WI); and miR-126 mimic and other chemicals were purchased from Sigma-Aldrich (St. Louis, MO).

\section{Cell culture}

The human prostate cancer cell lines (PC3 and DU145) were purchased from American Type Culture Collection (Manassas, VA). Human primary osteoblasts were obtained from Lonza (Walkersville, MD). Cells were maintained at $37^{\circ} \mathrm{C}$ in a $5 \% \mathrm{CO} 2$ atmosphere in RPMI-1640 medium supplemented with $20 \mathrm{mM}$ HEPES, $10 \%$ heat-inactivated FBS, $2 \mathrm{mM}$ glutamine, $100 \mathrm{U} / \mathrm{mL}$ penicillin, and $100 \mu \mathrm{g} / \mathrm{mL}$ streptomycin (Invitrogen;
Carlsbad, CA). To obtain osteoblast-conditioned medium (OBCM), cells were grown to confluence and culture media were changed to RPMI without FBS. OBCM was collected two days after the medium change and stored at $-70^{\circ} \mathrm{C}$ until use. In serial experiments, osteoblasts were transfected for $24 \mathrm{~h}$ with WISP-1 or control shRNA to prevent WISP-1 production and the medium was collected $48 \mathrm{~h}$ later. The level of WISP-1 in the culture medium was assayed with a WISP-1 enzyme-linked immunosorbent assay (ELISA) kit (R\&D Systems; Minneapolis, MN), as per the manufacturer's instructions.

\section{Migration and invasion assay}

Transwell inserts (Costar; New York, NY; pore size, $8 \mu \mathrm{m})$ in 24-well dishes were used for the migration assay. For invasion assays, filters were pre-coated with $30 \mu \mathrm{L}$ Matrigel basement membrane matrix (BD Biosciences; Bedford, MA) for $30 \mathrm{~min}$. Procedures were similar for the migration and invasion assays. Before the migration assay, cells were pretreated for $30 \mathrm{~min}$ with inhibitors (FAK inhibitor, SB203580, or vehicle control [0.1\% dimethyl sulfoxide]), and $200 \mu \mathrm{L}$ serum-free medium containing approximately $1 \times 10^{4}$ cells was placed in the upper chamber, while $300 \mu \mathrm{L}$ serum-free medium containing WISP-1 or OBCM was added to the lower chamber. Plates were incubated for $16 \mathrm{~h}$ at $37^{\circ} \mathrm{C}$ in $5 \% \mathrm{CO} 2$, and cells were fixed in $3.7 \%$ formaldehyde solution for 15 min and stained with $0.05 \%$ crystal violet in phosphatebuffered saline (PBS) for $30 \mathrm{~min}$. Cells on the upper side of filters were removed with cotton-tipped swabs and the filters were washed with PBS. Cells on the underside were examined and counted under a microscope. Each clone was plated in triplicate for each experiment, which was repeated at least three times.

\section{Quantitative real-time polymerase chain reaction (PCR)}

Total RNA was extracted from cells using the TRIzol kit (MDBio Inc; Taipei, Taiwan). Reverse transcription reactions were performed, in which $2 \mu \mathrm{g}$ total RNA was reverse transcribed into cDNA using oligo (dT) primer. Quantitative real-time PCR (RTqPCR) was conducted with the TaqMan ${ }^{\circledR}$ one-step PCR Master Mix (Applied Biosystems; Foster City, CA). Total complementary DNA (100 $\mathrm{ng} / 25-\mu \mathrm{L}$ reaction) was mixed with sequence-specific primers and TaqMan ${ }^{\circledR}$ probes, as per the manufacturer's instructions; sequences for all target gene primers and probes were purchased commercially (Applied Biosystems), and $\beta$-actin served as an internal control. q-PCR assays were carried out in triplicate, using a StepOnePlus sequence detection system. Cycling conditions were $10 \mathrm{~min}$ of polymerase activation at $95^{\circ} \mathrm{C}$, followed by 40 cycles at $95^{\circ} \mathrm{C}$ for 
$15 \mathrm{~s}$ and $60^{\circ} \mathrm{C}$ for $60 \mathrm{~s}$. Mir-X $\mathrm{X}^{\mathrm{TM}}$ miRNA First-Strand Synthesis and SYBR ${ }^{\circledR}$ RT-qPCR (Clontech Laboratories, Inc.; Mountain View, CA) kits were used for microRNA (miRNA) detection and reverse transcription, respectively. U6 snRNA levels served for normalization. A specific forward primer 5'-TCGTACCGTGAGTAATAATGCG-3' was used for miR-126. Forward and reverse primers for U6 were 5'-CTCGCTTCGGCAGCACATATACTA-3' and 5'-ACGAATTTGCGTGTCATCCTTGCG-3'. The threshold was set above the non-template control background and within the linear phase of target gene amplification to calculate the cycle number at which the transcript was detected (denoted as CT).

\section{Western blot analysis}

Cellular lysates were prepared as described previously [27] while proteins were resolved by sodium dodecyl sulfate-polyacrylamide gel electrophoresis and transferred onto Immobilon polyvinyldifluoride membranes. Blots were blocked with $4 \%$ bovine serum albumin for $1 \mathrm{~h}$ at room temperature and probed with rabbit anti-human antibodies against $\beta$-actin, VCAM1, p-p38, p38, p-JNK, JNK, p-FAK, FAK, p-ERK, or ERK (1:3000) for $2 \mathrm{~h}$ at room temperature. After three washings, blots were incubated with a donkey anti-rabbit peroxidase-conjugated secondary antibody (1:5000) for $1 \mathrm{~h}$ at room temperature. Blots were visualized using enhanced chemiluminescence and Kodak X-OMAT LS film (Eastman Kodak; Rochester, NY).

\section{Small interfering RNA (siRNA) transfection}

The siRNAs against human FAK, p38, VCAM1, and control siRNA were purchased from Santa Cruz Biotechnology. Cells were grown to $80 \%$ confluence in 6 -well plates and then transfected with siRNAs (100 nM) by Lipofectamine 2000 Transfection Reagent (Invitrogen; Carlsbad, CA) for $24 \mathrm{~h}$.

\section{Reporter gene assay}

To construct miR-126 promoter-luciferase and $V C A M-1$ 3'untranslated region (UTR)-luciferase plasmid, the $1.7-\mathrm{kb}$ miR-126 promoter and VCAM-1 3'UTR fragments containing the miR-126-binding site GTATAGTACTGGCATGGTACGG were inserted into the multiple cloning site of pGL2-Basic vector containing a luciferase reporter gene. All constructs were sequenced and verified. Cells grown to $80 \%$ confluence in 12 -well plates were transfected with $1 \mu \mathrm{g}$ luciferase plasmid using Lipofectamine 2000. To prepare lysates, $100 \mu \mathrm{L}$ reporter lysis buffer (Promega) was added to each well and cells were scraped from the dishes. The supernatant was collected after centrifugation at 13,000 rpm for $2 \mathrm{~min}$. Aliquots of cell lysates $(20 \mu \mathrm{L})$ containing equal amounts of protein $(20-30 \mu \mathrm{g})$ were placed in wells of an opaque, black, 96-well plate; $80 \mu \mathrm{L}$ luciferase substrate was added to all samples, and luminescence was measured in a microplate luminometer.

\section{Statistics}

Data are presented as mean \pm standard error of mean (SEM). The Student's t test was used for statistical analysis between samples. Comparison of more than two groups was performed using one-way ANOVA with Bonferroni's post-hoc test, where $p<0.05$ was considered significant.

\section{ACKNOWLEDGMENTS}

This work was supported by grants from the National Science Council of Taiwan (NSC100-2320-B039-028-MY3, NSC102-2632-B-039-001-MY3, NSC1012320-B-715-002-MY3, NSC102-2314-B-002-163-MY2).

\section{Conflict of Interest statement}

All authors have no financial or personal relationships with other people or organizations that could inappropriately influence our work.

\section{REFERENCES}

1. Bryant RJ and Hamdy FC. Screening for prostate cancer: an update. European urology. 2008; 53(1):37-44.

2. Mundy GR. Metastasis to bone: causes, consequences and therapeutic opportunities. Nature reviews Cancer. 2002; 2(8):584-593.

3. Shah RB, Mehra R, Chinnaiyan AM, Shen R, Ghosh D, Zhou M, Macvicar GR, Varambally S, Harwood J, Bismar TA, Kim R, Rubin MA and Pienta KJ. Androgenindependent prostate cancer is a heterogeneous group of diseases: lessons from a rapid autopsy program. Cancer research. 2004; 64(24):9209-9216.

4. Hanahan D and Weinberg RA. The hallmarks of cancer. Cell. 2000; 100(1):57-70.

5. Ell B and Kang Y. SnapShot: Bone Metastasis. Cell. 2012; 151(3):690-690 e691.

6. Kristensen HB, Andersen TL, Marcussen N, Rolighed L and Delaisse JM. Osteoblast Recruitment Routes in Human Cancellous Bone Remodeling. The American journal of pathology. 2014.

7. Weilbaecher KN, Guise TA and McCauley LK. Cancer to bone: a fatal attraction. Nature reviews Cancer. 2011; 11(6):411-425.

8. Hsu YL, Hou MF, Kuo PL, Huang YF and Tsai EM. Breast 
tumor-associated osteoblast-derived CXCL5 increases cancer progression by ERK/MSK1/Elk-1/snail signaling pathway. Oncogene. 2013; 32(37):4436-4447.

9. Hsu YL, Huang MS, Yang CJ, Hung JY, Wu LY and Kuo PL. Lung tumor-associated osteoblast-derived bone morphogenetic protein-2 increased epithelial-tomesenchymal transition of cancer by Runx2/Snail signaling pathway. The Journal of biological chemistry. 2011; 286(43):37335-37346.

10. Holbourn KP, Acharya KR and Perbal B. The CCN family of proteins: structure-function relationships. Trends in biochemical sciences. 2008; 33(10):461-473.

11. French DM, Kaul RJ, D'Souza AL, Crowley CW, Bao M, Frantz GD, Filvaroff EH and Desnoyers L. WISP1 is an osteoblastic regulator expressed during skeletal development and fracture repair. The American journal of pathology. 2004; 165(3):855-867.

12. Chen PP, Li WJ, Wang Y, Zhao S, Li DY, Feng LY, Shi XL, Koeffler HP, Tong XJ and Xie D. Expression of Cyr61, CTGF, and WISP-1 correlates with clinical features of lung cancer. PloS one. 2007; 2(6):e534.

13. Hou CH, Chiang YC, Fong YC and Tang CH. WISP-1 increases MMP-2 expression and cell motility in human chondrosarcoma cells. Biochemical pharmacology. 2011; 81(11):1286-1295.

14. Nagai $Y$, Watanabe M, Ishikawa S, Karashima R, Kurashige J, Iwagami S, Iwatsuki M, Baba Y, Imamura Y, Hayashi N and Baba H. Clinical significance of Wnt-induced secreted protein-1 (WISP-1/CCN4) in esophageal squamous cell carcinoma. Anticancer research. 2011; 31(3):991-997.

15. Pennica D, Swanson TA, Welsh JW, Roy MA, Lawrence DA, Lee J, Brush J, Taneyhill LA, Deuel B, Lew M, Watanabe C, Cohen RL, Melhem MF, Finley GG, Quirke $\mathrm{P}$, Goddard AD, et al. WISP genes are members of the connective tissue growth factor family that are up-regulated in wnt-1-transformed cells and aberrantly expressed in human colon tumors. Proc Natl Acad Sci U S A. 1998; 95(25):14717-14722.

16. Xie D, Yin D, Wang HJ, Liu GT, Elashoff R, Black K and Koeffler HP. Levels of expression of CYR61 and CTGF are prognostic for tumor progression and survival of individuals with gliomas. Clin Cancer Res. 2004; 10(6):2072-2081.

17. Chuang JY, Chang AC, Chiang IP, Tsai MH and Tang CH. Apoptosis signal-regulating kinase 1 is involved in WISP1 -promoted cell motility in human oral squamous cell carcinoma cells. PloS one. 2013; 8(10):e78022.

18. Makrilia N, Kollias A, Manolopoulos L and Syrigos K. Cell adhesion molecules: role and clinical significance in cancer. Cancer investigation. 2009; 27(10):1023-1037.

19. Desgrosellier JS and Cheresh DA. Integrins in cancer: biological implications and therapeutic opportunities. Nature reviews Cancer. 2010; 10(1):9-22.

20. Denys A, Clavel G, Semerano L, Lemeiter D and Boissier MC. A1.6 Vascular adhesion molecule VCAM-1 overexpression in collagen induced arthritis : a model for rheumatoid arthritis vascular dysfunction. Annals of the rheumatic diseases. 2014; 73 Suppl 1:A3.

21. Ding YB, Chen GY, Xia JG, Zang XW, Yang HY and Yang L. Association of VCAM-1 overexpression with oncogenesis, tumor angiogenesis and metastasis of gastric carcinoma. World J Gastroenterol. 2003; 9(7):1409-1414.

22. Lin KY, Lu D, Hung CF, Peng S, Huang L, Jie C, Murillo F, Rowley J, Tsai YC, He L, Kim DJ, Jaffee E, Pardoll D and $\mathrm{Wu}$ TC. Ectopic expression of vascular cell adhesion molecule-1 as a new mechanism for tumor immune evasion. Cancer research. 2007; 67(4):1832-1841.

23. Martinez LM, Vallone VB, Labovsky V, Choi H, Hofer EL, Feldman L, Bordenave RH, Batagelj E, Dimase F, Villafane $\mathrm{AR}$ and Chasseing NA. Changes in the peripheral blood and bone marrow from untreated advanced breast cancer patients that are associated with the establishment of bone metastases. Clinical \& experimental metastasis. 2014; 31(2):213-232.

24. Joo YN, Eun SY, Park SW, Lee JH, Chang KC and Kim HJ. Honokiol inhibits U87MG human glioblastoma cell invasion through endothelial cells by regulating membrane permeability and the epithelial-mesenchymal transition. International journal of oncology. 2014; 44(1):187-194.

25. Lu X, Mu E, Wei Y, Riethdorf S, Yang Q, Yuan M, Yan J, Hua Y, Tiede BJ, Haffty BG, Pantel K, Massague J and Kang Y. VCAM-1 promotes osteolytic expansion of indolent bone micrometastasis of breast cancer by engaging alpha4beta1-positive osteoclast progenitors. Cancer cell. 2011; 20(6):701-714.

26. Chen PC, Cheng HC, Yang SF, Lin CW and Tang CH. The $\mathrm{CCN}$ family proteins: modulators of bone development and novel targets in bone-associated tumors. Biomed Res Int. 2014; 2014:437096.

27. Huang CY, Chen SY, Tsai HC, Hsu HC and Tang CH. Thrombin induces epidermal growth factor receptor transactivation and CCL2 expression in human osteoblasts. Arthritis Rheum. 2012; 64(10):3344-3354.

28. Chirgwin JM and Guise TA. Molecular mechanisms of tumor-bone interactions in osteolytic metastases. Critical reviews in eukaryotic gene expression. 2000; 10(2):159178.

29. Ono M, Inkson CA, Kilts TM and Young MF. WISP-1/ CCN4 regulates osteogenesis by enhancing BMP-2 activity. Journal of bone and mineral research : the official journal of the American Society for Bone and Mineral Research. 2011; 26(1):193-208.

30. Brigstock DR. The CCN family: a new stimulus package. J Endocrinol. 2003; 178(2):169-175.

31. Guan JL. Role of focal adhesion kinase in integrin signaling. The international journal of biochemistry \& cell biology. 1997; 29(8-9):1085-1096.

32. Hour MJ, Tsai SC, Wu HC, Lin MW, Chung JG, Wu JB, Chiang JH, Tsuzuki $\mathrm{M}$ and Yang JS. Antitumor 
effects of the novel quinazolinone MJ-33: inhibition of metastasis through the MAPK, AKT, NF-kappaB and AP-1 signaling pathways in DU145 human prostate cancer cells. International journal of oncology. 2012; 41(4):1513-1519.

33. Banerjee N, Kim H, Talcott S and Mertens-Talcott S. Pomegranate polyphenolics suppressed azoxymethaneinduced colorectal aberrant crypt foci and inflammation: possible role of miR-126/VCAM-1 and miR-126/PI3K/ AKT/mTOR. Carcinogenesis. 2013; 34(12):2814-2822.

34. Sun C, Alkhoury K, Wang YI, Foster GA, Radecke CE, Tam K, Edwards CM, Facciotti MT, Armstrong EJ, Knowlton AA, Newman JW, Passerini AG and Simon SI. IRF-1 and miRNA126 modulate VCAM-1 expression in response to a high-fat meal. Circulation research. 2012; 111(8):1054-1064.

35. Harris TA, Yamakuchi M, Ferlito M, Mendell JT and Lowenstein CJ. MicroRNA-126 regulates endothelial expression of vascular cell adhesion molecule 1 . Proceedings of the National Academy of Sciences of the United States of America. 2008; 105(5):1516-1521.

36. Del Conte A, Minatel E, Schinella D, Baresic T, Basso SM and Lumachi F. Complete Metabolic Remission with Gefitinib in a Hemodialysis Patient with Bone Metastases from Non-small Cell Lung Cancer. Anticancer research. 2014; 34(1):319-322.

37. Ell B, Mercatali L, Ibrahim T, Campbell N, Schwarzenbach H, Pantel K, Amadori D and Kang Y. Tumor-induced osteoclast miRNA changes as regulators and biomarkers of osteolytic bone metastasis. Cancer cell. 2013; 24(4):542556.

38. Ricci E, Mattei E, Dumontet C, Eaton CL, Hamdy F, van der Pluije G, Cecchini M, Thalmann G, Clezardin P and Colombel M. Increased expression of putative cancer stem cell markers in the bone marrow of prostate cancer patients is associated with bone metastasis progression. The Prostate. 2013; 73(16):1738-1746.

39. Itatani Y, Kawada K, Fujishita T, Kakizaki F, Hirai H, Matsumoto T, Iwamoto M, Inamoto S, Hatano E, Hasegawa S, Maekawa T, Uemoto S, Sakai Y and Taketo MM. Loss of SMAD4 from colorectal cancer cells promotes CCL15 expression to recruit CCR1+ myeloid cells and facilitate liver metastasis. Gastroenterology. 2013; 145(5):1064-1075 e1011.

40. Desgrosellier JS and Cheresh DA. Integrins in cancer: biological implications and therapeutic opportunities. Nat Rev Cancer. 10(1):9-22.

41. Esquela-Kerscher A and Slack FJ. Oncomirs - microRNAs with a role in cancer. Nature reviews Cancer. 2006; 6(4):259-269.

42. Zhang Quan JK-T. Long noncoding RNAs and viral infections. BioMedicine. 2013; 3(1):34-42.

43. Chen PS, Su JL and Hung MC. Dysregulation of microRNAs in cancer. J Biomed Sci. 2012; 19:90.

44. Kawaki H, Kubota S, Suzuki A, Suzuki M, Kohsaka K,
Hoshi K, Fujii T, Lazar N, Ohgawara T, Maeda T, Perbal B, Takano-Yamamoto T and Takigawa M. Differential roles of CCN family proteins during osteoblast differentiation: Involvement of Smad and MAPK signaling pathways. Bone. 2011; 49(5):975-989. 